\title{
A theoretical analysis of price elasticity of energy demand in multi-stage energy conversion systems.
}

\author{
Robert Lowe
}

Centre for the Built Environment, Leeds Metropolitan University, UK

February $2003^{1}$

\begin{abstract}
The objective of this paper is an analytical exploration of the problem of price elasticity of energy demand in multi-stage energy conversion systems. The paper describes in some detail an analytical model of energy demand in such systems. Under a clearly stated set of assumptions, the model makes it possible to explore both the impacts of the number of sub-systems, and of varying sub-system elasticities on overall system elasticity. The analysis suggests that overall price elasticity of energy demand for such systems will tend asymptotically to unity as the number of sub-systems increases.
\end{abstract}

Keywords: $\quad$ price elasticity, analytical model, multi-stage systems

\section{Introduction}

This paper has been written in an attempt to understand certain aspects of the impact of energy price on demand in multi-stage energy conversion systems. For a variety of reasons ranging from problems of time lags and short time series, to the problem of non-stationarity, any analytical treatment of such systems is unlikely to give more than a rather incomplete picture of their behaviour. To make progress at all, the author has had to assume that:

- time lags can be neglected

- the performance of each sub-system depends only on the price of energy immediately upstream

- the additional costs imposed by each sub-system relate only to the energy dissipated by that sub-system

- the performance of each sub-system is reversible, and that it can be represented analytically by a power law. ${ }^{2}$

The nature of the energy conversion system is sketched in Figure 1. 
The nomenclature used to describe this system is as follows:

$E_{i} \quad$ is the energy flux following the $j$ th stage of energy conversion

$\eta_{i} \quad$ is the $j$ th sub-system efficiency

$\eta_{i}^{\prime} \quad$ is the $j$ th sub-system efficiency in the case that up-stream subsystems are inelastic

$\alpha_{i} \quad$ is the $j$ th sub-system exponent of demand ${ }^{3}$, with respect to the effective cost of energy following the $(i-1)$ th stage of conversion

$\alpha_{\text {system }} \quad$ is the overall price elasticity of energy demand for the whole energy conversion system

$\alpha_{i, \text { effective }}$ is the overall price elasticity of energy demand for the whole energy conversion system

$c_{i} \quad$ is the effective cost of energy following the $i$ th energy conversion stage

$c_{0} \quad$ is the cost of primary energy, up-stream of all energy conversion stages

$c_{i, \text { base }} \quad$ is the effective value of $c_{i}$ in the base case, when $c_{0}=c_{0, \text { base }}$

As noted above, the $j$ th sub-system efficiency is assumed to depend on the effective cost of energy following the $(i-1)$ th stage of energy conversion. Thus:

$\eta_{i}=\eta_{i, \text { base }} \cdot\left(c_{i-1} / c_{i-1, \text { base }}\right)^{\alpha_{i}}$

and

$c_{n+1}=\frac{c_{n}}{\eta_{n+1}}=\frac{c_{0}}{\prod_{i=1}^{n+1} \eta_{i}}$

\section{Evaluation of energy costs.}

Since the conversion efficiency of each sub-system is assumed to depend on the upstream energy price, the first stage in the process of analysis is to calculate these prices in terms of sub-system elasticities and primary energy price, $c_{0}$. We can then calculate the corresponding energy fluxes. For a single stage system:

$c_{1}=\frac{c_{0}}{\eta_{1}}=\frac{c_{0}}{\eta_{1, \text { base }} \cdot\left(c_{0} / c_{0, \text { base }}\right)^{\alpha_{i}}}$

and re-arranging: 
$c_{1} / c_{0, \text { base }}=\frac{\left(c_{0} / c_{0, \text { base }}\right)^{\left(1-\alpha_{1}\right)}}{\eta_{1, \text { base }}}$

For a 2-stage system:

$c_{2} / c_{0, \text { base }}=\frac{c_{1} / c_{0, \text { base }}}{\eta_{2}}=\frac{\left(c_{0} / c_{0, \text { base }}\right)^{\left(1-\alpha_{1}\right)}}{\eta_{1, \text { base }} \cdot \eta_{2, \text { base }} \cdot\left(c_{1} / c_{1, \text { base }}\right)^{\alpha_{2}}}$

but from equation 1 :

$c_{1, \text { base }}=c_{0, \text { base }} / \eta_{1, \text { base }}$

so:

$c_{2} / c_{0, \text { base }}=\frac{\left(c_{0} / c_{0, \text { base }}\right)^{\left(1-\alpha_{1}\right)}}{\eta_{1, \text { base }} \cdot \eta_{2, \text { base }} \cdot\left(\eta_{1, \text { base }} \cdot c_{1} / c_{0, \text { base }}\right)^{\alpha_{2}}}$

Substituting for $\eta_{1, \text { base }} \cdot c_{1} / c_{0, \text { base }}$ from equation (4):

$c_{2} / c_{0, \text { base }}=\frac{\left(c_{0} / c_{0, \text { base }}\right)^{\left(1-\alpha_{1}\right)}}{\eta_{1, \text { base }} \cdot \eta_{2, \text { base }} \cdot\left(c_{0} / c_{0, \text { base }}\right)^{\left(1-\alpha_{1}\right) \cdot \alpha_{2}}}$

and re-arranging:

$c_{2} / c_{0, \text { base }}=\frac{\left(c_{0} / c_{0, \text { base }}\right)^{\left(1-\alpha_{1}\right)\left(1-\alpha_{2}\right)}}{\eta_{1, \text { base }} \cdot \eta_{2, \text { base }}}$

In general (a formal proof is presented in an appendix):

$c_{n} / c_{0, \text { base }}=\frac{\left(c_{0} / c_{0, \text { base }}\right) \prod_{i=1}^{n}\left(1-\alpha_{i}\right)}{\prod_{i=1}^{n} \eta_{i, \text { base }}}$

\section{Evaluation of energy fluxes.}

Conceptually, the evaluation of energy fluxes is done in the opposite direction from the evaluation of prices. We assume that the energy flux from the final stage of conversion is fixed. Our objective is to calculate the input of primary energy that is needed to obtain this fixed quantity, under differing assumptions about the price of primary energy. For a single stage system: 
$E_{0}=\frac{E_{1}}{\eta_{1}}=\frac{E_{1}}{\eta_{1, \text { base }} \cdot\left(c_{0} / c_{0, \text { base }}\right)^{\alpha_{1}}}$

and since $E_{0, \text { base }}=E_{1} / \eta_{1, \text { base }}$

$$
E_{0}=E_{0, \text { base }} \cdot\left(c_{0} / c_{0, \text { base }}\right)^{-\alpha_{1}}
$$

which, for reasons that will become apparent, we will write:

$$
E_{0}=E_{0, \text { base }} \cdot\left(c_{0} / c_{0, \text { base }}\right)^{1-\left(1-\alpha_{1}\right)}
$$

For a two-stage system:

$$
E_{0}=\frac{E_{2}}{\eta_{1} \cdot \eta_{2}}
$$

Using equation 1 to expand $\eta_{1}$ and $\eta_{2}$ :

$$
\begin{aligned}
& E_{0}=\frac{E_{2}}{\eta_{1, \text { base }} \cdot\left(c_{0} / c_{0, \text { base }}\right)^{\alpha_{1}} \cdot \eta_{2, \text { base }} \cdot\left(c_{1} / c_{1, \text { base }}\right)^{\alpha_{2}}} \\
& \text { and since } E_{0, \text { base }}=\frac{E_{2}}{\eta_{1, \text { base }} \cdot \eta_{1, \text { base }}} \\
& E_{0}=\frac{E_{0, \text { base }}}{\left(c_{0} / c_{0, \text { base }}\right)^{\alpha_{1}} \cdot\left(c_{1} / c_{1, \text { base }}\right)^{\alpha_{2}}}
\end{aligned}
$$

But from equation 2, $c_{1, \text { base }}=c_{0, \text { base }} / \eta_{1 \text {, base }}$

$$
E_{0}=\frac{E_{0, \text { base }}}{\left(c_{0} / c_{0, \text { base }}\right)^{\alpha_{1}} \cdot\left(\eta_{1, \text { base }} \cdot c_{1} / c_{0, \text { base }}\right)^{\alpha_{2}}}
$$

Substituting from equation 4 and simplifying:

$$
E_{0}=E_{0, \text { base }} \cdot\left(c_{0, \text { base }} / c_{0}\right)^{1-\left(1-\alpha_{1}\right) \cdot\left(1-\alpha_{2}\right)}
$$

For an n-stage system (again, a formal proof is presented in an appendix):

$$
E_{0}=E_{0, \text { base }} \cdot\left(c_{0, \text { base }} / c_{0}\right)^{1-\prod_{i=1}^{n}\left(1-\alpha_{i}\right)}
$$

The overall system elasticity is given by: 
$\alpha_{\text {system }}=1-\prod_{i=1}^{n}\left(1-\alpha_{i}\right)$

In the special case that all sub-system elasticities are equal, that is when $\alpha_{i}=\alpha$, the overall system elasticity reduces to:

$\alpha_{\text {system }}=1-(1-\alpha)^{n}$

When sub-system elasticities are small, that is $\sum \alpha_{i}<<1$, the overall system elasticity approximates to the sum of the sub-system elasticities.

$\alpha_{\text {system }} \approx \sum_{i=1}^{n} \alpha_{i}-\mathrm{O}\left(\alpha_{j} \cdot \alpha_{k}\right)$

\section{Discussion and Conclusions.}

For a system in which sub-systems are not price elastic, $\alpha_{i}=0$, and equation 19 simplifies to:

$E_{0}=E_{0, \text { base }}$

For a completely elastic system, $\alpha_{i}=1$, and equation 2 simplifies to:

$$
E_{0}=E_{0, \text { base }} \cdot\left(c_{0, \text { base }} / c_{0}\right)
$$

In such a system, energy use is inversely proportional to energy price. More importantly, equations 20 and 21 suggest a tendency for overall system elasticity of complex, multi-stage systems to tend to unity, even where all $\alpha_{i}<1$. This key result can be seen more clearly when all partial elasticities are equal: $\alpha_{i}=\alpha$ for all $i$. In this case the overall elasticity, given by equation 21 , is an exponential function of the number of stages, $n$. This function is plotted for $\alpha=0.25$ and $\alpha=0.5$ in Figure 2 .

In practice, all stages in a multi-stage system may not be able to respond immediately to a change in upstream energy price. Possible reasons for this include long response times associated with long physical lifetimes of particular pieces of infrastructure such as power stations, and hysteresis induced by network effects. Where upstream stages do not respond quickly or at all, the effect is to induce a larger response from downstream stages, coupled with reduced overall response. The cost of the larger short-term response from downstream stages is likely to be short-term over-investment in these stages, coupled with a tendency for the response of the whole system to overshoot in the long term.

This can be illustrated as follows. If all sub-systems respond to an energy price change, then the efficiency of sub-system $i$ is given by equation 1 : 


$$
\begin{aligned}
\eta_{i} & =\eta_{i, \text { base }} \cdot\left(c_{i-1} / c_{i-1, \text { base }}\right)^{\alpha_{i}} \\
& =\eta_{i, \text { base }} \cdot\left(\left(c_{i-1} / c_{0}\right) \cdot \prod_{j=1}^{i-1} \eta_{j}\right)^{\alpha_{i}}
\end{aligned}
$$

and substituting for $\left(c_{i-1} / c_{0}\right) \cdot \prod_{j=1}^{i-1} \eta_{j}$ from equation 10 :

$$
\eta_{i}=\eta_{i, \text { base }} \cdot\left(c_{0} / c_{0, \text { base }}\right)^{\alpha_{i}} \cdot \prod_{j=1}^{i-1}\left(1-\alpha_{j}\right)
$$

In this case, the effective elasticity of the $i$ th sub-system, with respect to changes in $c_{0}$ rather than $c_{i-1}$, is not $\alpha_{i}$ but:

$$
\alpha_{i, \text { effective }}=\alpha_{i} \cdot \prod_{j=1}^{i-1}\left(1-\alpha_{j}\right)
$$

The later any particular sub-system appears in the chain of conversion, the smaller will be its effective elasticity. Elastic upstream sub-systems attenuate the price signal experienced by downstream sub-systems and thus the response of those upstream sub-systems to an overall change in energy price. In the simple case that all sub-system elasticities are equal, this upstream shielding factor simplifies to an exponential function:

$$
(1-\alpha)^{i-1}
$$

The shielding effect of upstream sub-systems is illustrated in Figure 3 for $\alpha=0.5$. In a 2-stage system, with $\alpha_{1}=\alpha_{2}=0.5$, in which both stages are allowed to respond to a change in raw energy price, the effective elasticity of the second stage is $1 / 4$ rather than $1 / 2$ and the overall system elasticity is $3 / 4$. Though this is just one of several possible theoretical explanations for such phenomena, it is easy to see how shielding, combined with time lags in upstream sub-systems and asymmetric price responses (Gately, 1992; Walker \& Wirl, 1993) can give rise to empirical long-run system elasticities greater than unity.

If on the other hand we assume that only sub-system $i$ of an $n$-stage system is elastic, then the change in efficiency of sub-system $i$ is given by:

$$
\eta_{i}^{\prime}=\eta_{i, \text { base }} \cdot\left(c_{0} / c_{0, \text { base }}\right)^{\alpha_{i}}
$$

In this case the effective elasticity of the $i$ th sub-system is, as we would expect, simply $\alpha_{i}$. Thus, non-response of upstream stages increases the effective 
elasticity of the $i$ th sub-system with respect to changes in the price of raw energy, $c_{0}$, by a factor of:

$$
\prod_{j=1}^{i-1}\left(1-\alpha_{j}\right)
$$

and reduces the overall system elasticity by a factor of:

$$
\frac{1-\prod_{j=1}^{n}\left(1-\alpha_{j}\right)}{\alpha_{i}}
$$

compared with the case where all systems respond to changes in price. In the simple case that all sub-system elasticities are equal, the reduction in overall elasticity simplifies to:

$$
\left(1-(1-\alpha)^{n}\right) / \alpha
$$

The results of this paper may be of significance in that many practical energy conversion systems do in fact consist of chains of linked processes. Four simple examples within the built environment are:

- mechanical ventilation systems - inlet and exhaust resistance, motor efficiency, fan efficiency, distribution system, supply and extract terminals (Nørgård et al., 1983)

- lighting systems - electricity supply system, lamp, luminaire, lighting control system, building (Verderber \& Rubinstein, 1984; Ne'eman, 1984)

- space heating in buildings - energy supply system, space heating system, thermal envelope

- space cooling in buildings - energy supply system, space cooling system, thermal envelope

Lighting, heating, cooling and air movement account for most of the energy used in the built environment. Two further examples illustrate the potentially widespread applicability of this analysis:

- IT systems - electricity supply system, power supply, energy management systems, CPU, screen (Norford et al., 1989); and

- vehicles - oil refinery, engine, gearbox, transmission, vehicle mass and air resistance (von Weizsäcker et al., 1996)

A more detailed examination of these systems reveals many additional subsystems, but also structures that are significantly more complex than the simple chain of conversion that forms the conceptual basis for the analysis presented in this paper. Moreover, in practice, many aspects of these systems are not determined by micro-economic optimisation. For example, the thermal properties of building envelopes are substantially determined by regulation. Nevertheless, the demands of regulation are themselves influenced by economic analysis, and perceptions of future energy price (DETR, 2000). 
Despite these caveats, it would appear likely that technological advance and economic development generally lead to an increasing proportion of complex multi-stage energy conversion systems. The analysis presented here would lead one to expect total price elasticities of such systems to be larger than sub-system analyses would suggest, and to approach unity. Moreover, the analysis suggests that in attempting to predict or understand overall empirical elasticities of energy conversion systems, one should place at least as much weight on the structure of such systems, as on the details of any particular sub-system. It is not the author's intent to present a complete review of empirical work on price elasticity of energy demand in support of this contention, but some work suggests that this might indeed be the case. Von Weizsäcker \& Jesinghaus (1992) suggest a price elasticity for energy use in cars in the region of unity based on comparison of energy use and price data in 14 countries, Berkhout et al. (2000) state that the long term elasticity for passenger transport is in the range 0.8-1.0, while Goodwin (1992) gives a value of 1.2 for the long term price elasticity of energy demand for transport in the UK. In other areas such as lighting and IT, while a combination of short runs of data and non-stationarity make it difficult to confirm this empirically, engineering analyses suggest that elasticities should be high.

The analysis presented here does not apply directly to systems other than simple energy conversion chains. A thorough treatment of the problem of price elasticity of transport demand cannot, for example, avoid considering sub-systems such as social attitudes to cycling and walking, logistics strategies for freight distribution, urban density and form, and living and working patterns, none of which satisfies this condition. While it would be interesting to attempt to extend the argument presented here to a wider range of systems and to include cost categories other than energy, it may not be easy to do this analytically. Nevertheless there appears to be no obvious reason why the basic results of this paper should not apply, at least qualitatively, to such systems.

To conclude, the main policy implications of this paper are that:

- the overall structure of energy conversion systems may be at least as important in determining system behaviour as the details of any particular sub-system

- long run price elasticities for many energy conversion systems may approach unity $^{4}$

- pricing policies may therefore have a significant impact on energy demand and carbon emissions for many energy using systems, and

- the impact of such policies is likely to be maximised if energy or carbon taxation is levied at the earliest possible point in energy conversion chains.

\section{Acknowledgement}

The author wishes to thank Harry D. Saunders for his advice and encouragement during the writing of this paper. 


\section{$\underline{\text { References }}$}

Berkhout, P.H.G., Muskens, J.C. \& Welthuijsen, J.W., 2000. Defining the rebound effect. Energy Policy 28 425-432.

DETR (2000) Building Regulations. Proposals for Amending the Energy Efficiency Provisions. A Consultation Paper issued by the Building Regulations Divisision. London: DETR.

Gately, D., 1992. Imperfect price reversibility of US gasoline demand: asymmetric price responses to price increases and decreases. The Energy Journal 13 (4) 179-207.

Goodwin, P.B., 1992. A Review of New Demand Elasticities with Special Reference to Short and Long Run Effects on Price Changes. Journal of Transport Economics and Policy 26 (2) 155-169.

Lowe, R.J., Sturges, J.S. \& Hodgson, N.J., 1997. Energy Analysis and Optimal Insulation Thickness. In: Proc. $2^{\text {nd }}$ International Conference on Buildings and the Environment, vol 1 533-540. Paris: CSTB.

Ne'eman, E., 1984. A comprehensive approach to the integration of daylight and electric light in buildings. Energy and Buildings 6 97-108.

Norford, L., Rabl, A., Harris, J. \& Roturier, J., 1989. Electronic Office Equipment: The Impact of Market Trends and Technology on End-Use Demand for Electricity. In: Johansson, T.B., Bodlund, B. \& Williams, R.H. (Eds), Electricity: Efficient End-Use and New Generation Technologies and Their Planning Implications. Lund University Press, pp 427-460.

Nørgård, J.S. Holck, J. Mehlsen, K., 1983. Langsigtede tekniske muligheder for elbesparelse. DEMO Projekt, Danmarks Tekniske Højskole.

Verderber, R.R \& Rubinstein, F.M., 1984. Mutual impacts of lighting controls and daylighting applications. Energy and Buildings 6 133-140.

Walker, I.O. \& Wirl, F., 1993. Irreversible price-induced efficiency improvements: theory and empirical application to road transportation. The Energy Journal 14 (4) 183-205.

Weizsäcker E.U.von \& Jesinghaus, J., 1992. Ecological Tax Reform. London: Zed Books.

Weizsäcker, E.U. von, Lovins, A.B. \& Lovins, L.H., 1997. Factor Four: Doubling Wealth, Halving Resource Use. London: Earthscan. 


\section{Appendix}

To avoid overburdening an already lengthy argument with detail, equations describing costs and energy use in an n-stage system were stated in the body of this paper without proof (equations 10 and 19). The purpose of this appendix is to provide inductive proofs for these two statements.

From equations 2 and 10 we have:

$$
c_{n+1} / c_{0, \text { base }}=\frac{\left(c_{0} / c_{0, \text { base }}\right) \prod_{i=1}^{n}\left(1-\alpha_{i}\right)}{\eta_{n+1} \cdot \prod_{i=1}^{n} \eta_{i, \text { base }}}
$$

Using equation 1 to expand $\eta_{n+1}$, we get:

$$
c_{n+1} / c_{0, \text { base }}=\frac{\left(c_{0} / c_{0, \text { base }}\right) \prod_{i=1}^{n}\left(1-\alpha_{i}\right)}{\eta_{n+1, \text { base }} \cdot\left(c_{n} / c_{n, \text { base }}\right)^{\alpha_{n+1}} \cdot \prod_{i=1}^{n} \eta_{i, \text { base }}}
$$

From equation 2 we note that:

$$
\begin{gathered}
c_{n, \text { base }}=\frac{c_{0, \text { base }}}{\prod_{i=1}^{n} \eta_{i, \text { base }}} \\
c_{n+1} / c_{0, \text { base }}=\frac{\left(c_{0} / c_{0, \text { base }}\right) \prod_{i=1}^{n}\left(1-\alpha_{i}\right)}{\left(c_{n} / c_{0, \text { base }} \cdot \prod_{i=1}^{n} \eta_{i, \text { base }}\right)^{\alpha_{n+1}} \cdot \prod_{i=1}^{n+1} \eta_{i, \text { base }}}
\end{gathered}
$$

Substituting from equation 10 , we get:

$$
c_{n+1} / c_{0, \text { base }}=\frac{\left(c_{0} / c_{0, \text { base }}\right) \prod_{i=1}^{n}\left(1-\alpha_{i}\right)}{\left(c_{0} / c_{0, \text { base }}\right)^{\alpha_{n+1} \cdot \prod_{i=1}^{n}\left(1-\alpha_{i}\right)} \cdot \prod_{i=1}^{n+1} \eta_{i, \text { base }}}
$$

and simplifying:

$$
c_{n+1} / c_{0, \text { base }}=\frac{\left(c_{0} / c_{0, \text { base }}\right) \prod_{i=1}^{n+1}\left(1-\alpha_{i}\right)}{\prod_{i=1}^{n+1} \eta_{i, \text { base }}}
$$


Since we proved earlier that equation 10 holds for $n=1$ and 2 , it follows that it is valid for all $n$.

Equation 19 for the primary energy flux of an $n$-stage system can be derived as follows. By definition:

$$
E_{0}=\frac{E_{n}}{\prod_{i=1}^{n} \eta_{i}}
$$

Using equation 1 to expand $\eta_{i}$ gives:

$$
E_{0}=\frac{E_{n}}{\eta_{1, \text { base }} \cdot\left(c_{0} / c_{0, \text { base }}\right)^{\alpha_{1}} \cdot \eta_{2, \text { base }} \cdot\left(c_{0} / c_{0, \text { base }}\right)^{\alpha_{2}} \ldots \eta_{n, \text { base }} \cdot\left(c_{0} / c_{0, \text { base }}\right)^{\alpha_{n}}}
$$

and substituting for $E_{n}$, we have:

$$
E_{0}=\frac{E_{0, \text { base }}}{\prod_{i=1}^{n}\left(c_{i-1} / c_{i-1, \text { base }}\right)^{\alpha_{i}}}
$$

From equation 2:

$$
c_{i-1, \text { base }}=\frac{c_{0, \text { base }}}{\prod_{j=1}^{i-1} \eta_{j, \text { base }}}
$$

Hence:

$$
E_{0}=\frac{E_{0, \text { base }}}{\prod_{i=1}^{n}\left(\left(c_{i-1} / c_{0, \text { base }}\right) \cdot \prod_{j=1}^{i-1} \eta_{j, \text { base }}\right)^{\alpha_{i}}}
$$

Substituting from equation 10 :

$$
E_{0}=E_{0, \text { base }} \cdot \prod_{i=1}^{n}\left(\left(c_{0, \text { base }} / c_{0}\right)^{\alpha_{i}} \cdot \prod_{j=1}^{i-1}\left(1-\alpha_{j}\right)\right)
$$

The product in the body of this expression can be expanded as follows: 


$$
\prod_{i=1}^{n}(\ldots)=\left(c_{0, \text { base }} / c_{0}\right)^{\alpha_{1}} \cdot()^{\left(1-\alpha_{1}\right) \cdot \alpha_{2}} \cdot()^{\left(1-\alpha_{1}\right) \cdot\left(1-\alpha_{2}\right) \cdot \alpha_{3}} \ldots()^{\left(1-\alpha_{1}\right) \ldots\left(1-\alpha_{n-1}\right) \cdot \alpha_{n}}
$$

The first two terms on the left can be combined to give:

$$
\prod_{i=1}^{n}(\ldots)=\left(c_{0, \text { base }} / c_{0}\right)^{1-\left(1-\alpha_{1}\right) \cdot\left(1-\alpha_{2}\right)} \cdot()^{\left(1-\alpha_{1}\right) \cdot\left(1-\alpha_{2}\right) \cdot \alpha_{3}} \ldots()^{\left(1-\alpha_{1}\right) \ldots\left(1-\alpha_{n-1}\right) \cdot \alpha_{n}}
$$

The first pair of terms in A14 can be similarly combined. After $j$ such operations, we have:

$$
\prod_{i=1}^{n}(\ldots)=()^{1-\left(1-\alpha_{1}\right) \cdot\left(1-\alpha_{2}\right) \ldots\left(1-\alpha_{j}\right)} \cdot()^{1-\left(1-\alpha_{1}\right) \cdot\left(1-\alpha_{2}\right) \ldots\left(1-\alpha_{j+1}\right)} \ldots()^{\left(1-\alpha_{1}\right) \ldots\left(1-\alpha_{n-1}\right) \cdot \alpha_{n}}
$$

Combining the first two terms in equation 15, we get:

$$
\prod_{i=1}^{n}(\ldots)=()^{1-\left(1-\alpha_{1}\right) \cdot\left(1-\alpha_{2}\right) \ldots\left(1-\alpha_{j}\right) \cdot\left(1-\alpha_{j+1}\right)} \ldots()^{\left(1-\alpha_{1}\right) \ldots\left(1-\alpha_{n-1}\right) \cdot \alpha_{n}}
$$

Without more elaboration, it is obvious that all further terms can be combined to give the result presented earlier as equation 19:

$$
E_{0}=E_{0, \text { base }} \cdot\left(c_{0, \text { base }} / c_{0}\right)^{1-} \prod_{i=1}^{n}\left(1-\alpha_{i}\right)
$$


Figures

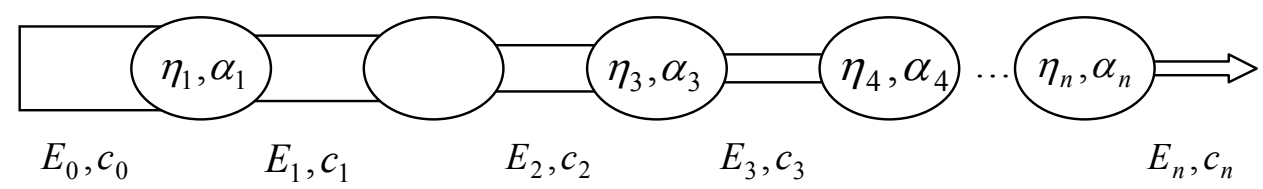

Figure 1. Components of multi-stage energy conversion system. 


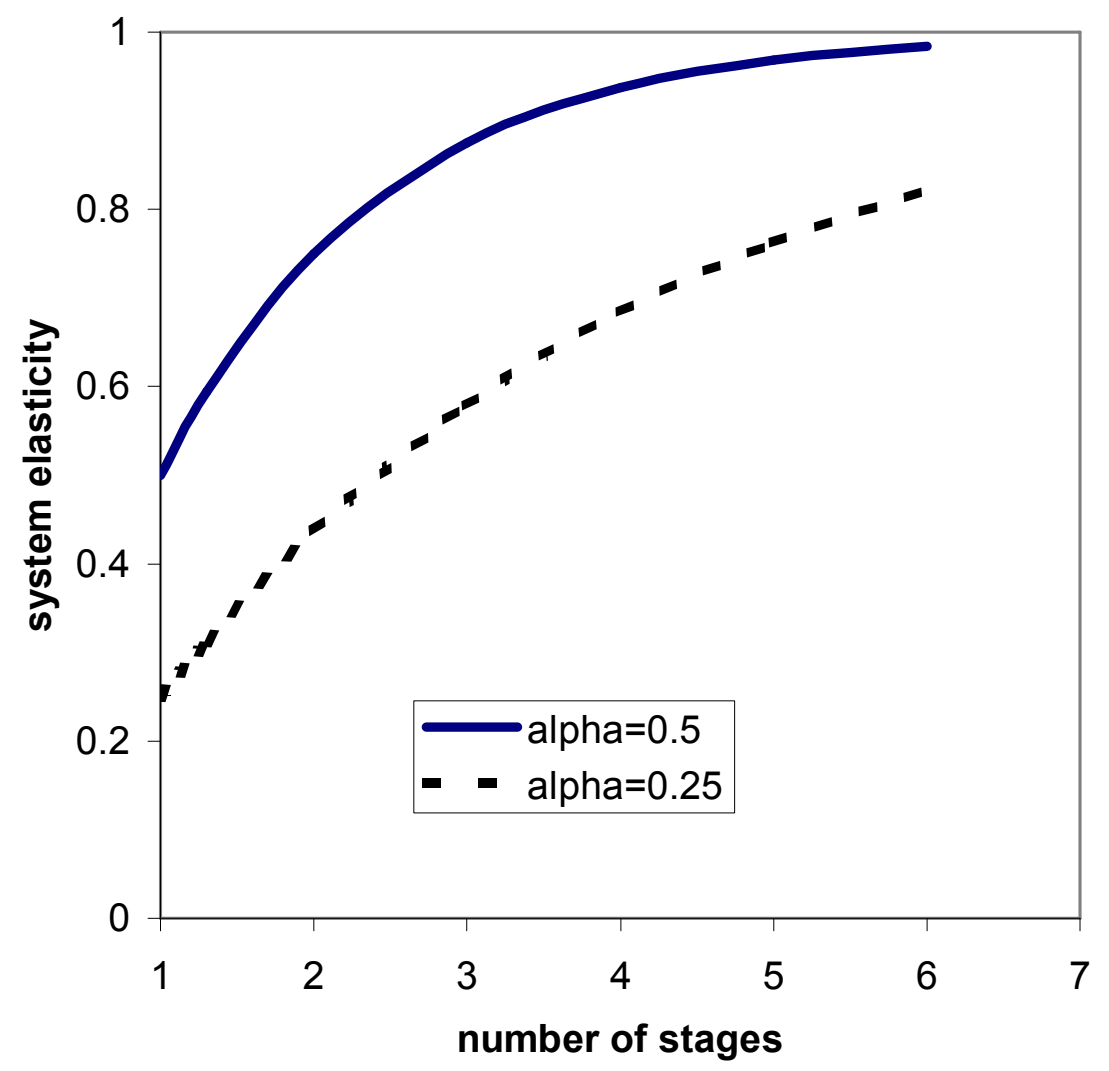

Figure 2. System elasticity versus number of sub-systems. 


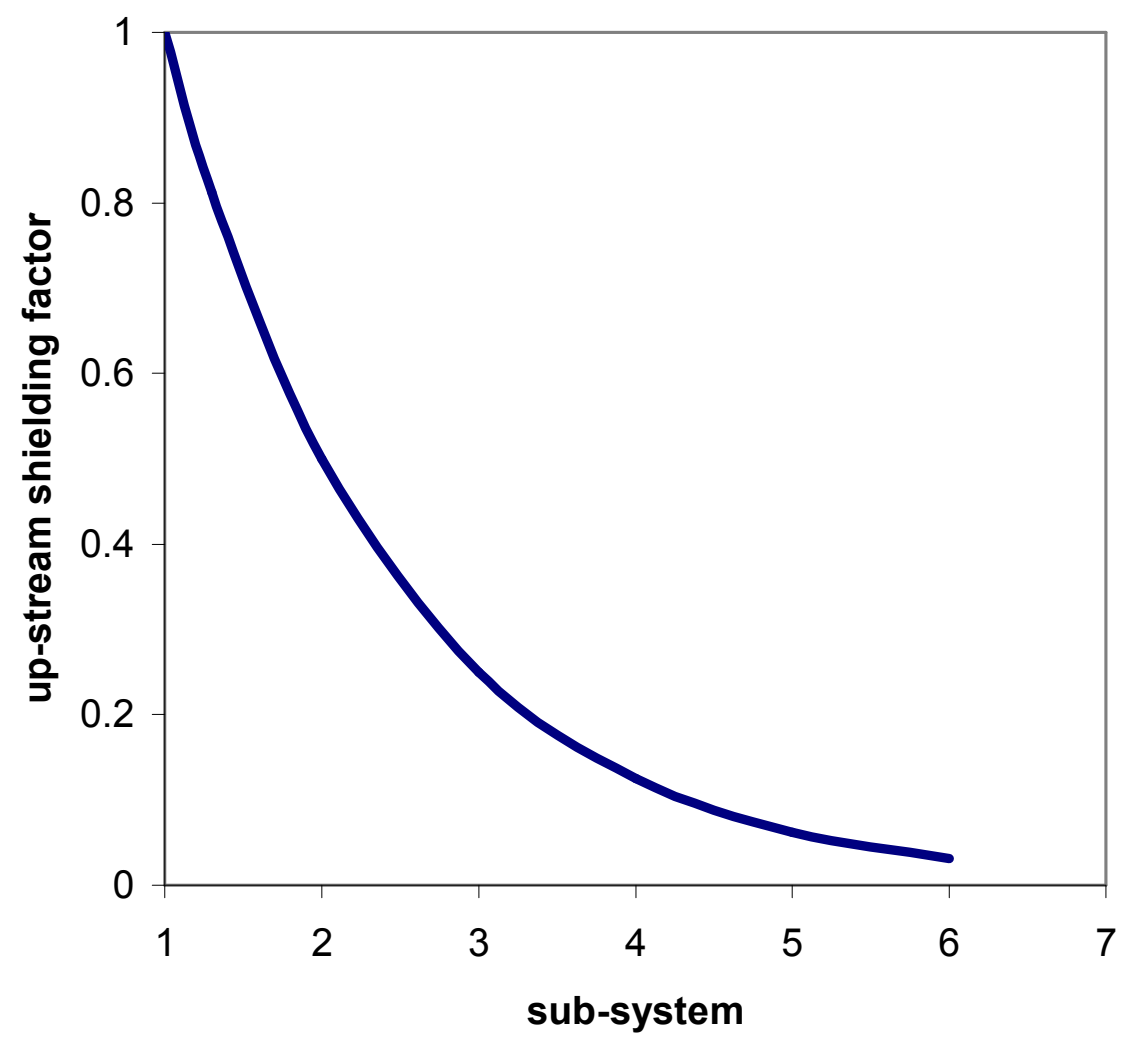

Figure 3. Shielding effect of up-stream sub-systems on effective elasticity of final sub-system in an $n$-stage energy conversion system. 


\section{Footnotes}

$1 \quad$ The first version of this paper was written in August 1998.

2 In certain simple cases, engineering analysis would lead one to expect such behaviour, and the expected exponents can be calculated. Space heating in buildings is one such case. The predicted price elasticity of space heating in buildings, based on re-optimisation of the building thermal envelope alone, is approximately 0.5 (Lowe et al 1997).

$3 \quad$ For small changes in energy cost, such that $c_{i-1} / c_{i-1, \text { base }} \approx 1$

$\left(1 / E_{i-1, \text { base }}\right) \cdot d E_{i-1} \approx \alpha_{i} \cdot\left(1 / c_{i-1, \text { base }}\right) \cdot d c_{i-1}$

Hence, the exponent, $\alpha_{i}$ is the price elasticity of the $i$ th sub-system with respect to the effective cost of energy from the preceding stages of conversion. It seems appropriate to refer to this exponent as a partial or sub-system elasticity of demand.

4 While this conclusion is not new, its derivation from the structures of energy consuming systems is original 